\title{
Derivation and validation of a multivariate model to predict mortality from pulmonary embolism with cancer: the POMPE-C tool
}

\author{
Jeffrey A. Kline ${ }^{1}$, Pierre-Marie Roy ${ }^{2}$, Martin P. Than ${ }^{3}$, Jackeline Hernandez ${ }^{1}$, D. Mark \\ Courtney $^{4}$, Alan E. Jones ${ }^{5}$, Andrea Penazola ${ }^{6}$, and Charles V. Pollack Jr. ${ }^{7}$ \\ ${ }^{1}$ Department of Emergency Medicine 1000 Blythe Boulevard MEB 3rd floor, Room 306 Charlotte, \\ NC $28203^{2}$ Emergency Department CHU Angers, Université d'Angers, France LUNAM \\ Université, Angers, France PMRoy@chu-angers.fr ${ }^{3}$ Christchurch Hospital, Christchurch, New \\ Zealand martin.than@cdhb.govt.nz ${ }^{4}$ Department of Emergency Medicine Feinberg School of \\ Medicine, Northwestern University, Chicago, IL. mcourtney@nmff.org 5epartment of Emergency \\ Medicine University of Mississippi Medical Center Jackson, MS aejones@umc.edu ${ }^{6}$ Andrea \\ Penaloza, MD Emergency Department Cliniques Universitaires St-Luc 10 Av. Hippocrate 1200 \\ Brussels Belgium andrea.penaloza@uclouvain.be ${ }^{7}$ Department of Emergency Medicine \\ Pennsylvania Hospital University of Pennsylvania Health System 800 Spruce St Philadelphia, PA \\ 19107 cvpollack@gmail.com
}

\section{Abstract \\ Background-Clinical guidelines recommend risk stratification of patients with acute pulmonary embolism (PE). Active cancer increases risk of PE and worsens prognosis, but also causes incidental PE that may be discovered during cancer staging. No quantitative decision instrument has been derived specifically for patients with active cancer and PE.}

Methods-Classification and regression technique was used to reduce 25 variables prospectively collected from 408 patients with $\mathrm{AC}$ and $\mathrm{PE}$. Selected variables were transformed into a logistic regression model, termed POMPE-C, and compared with the pulmonary embolism severity index (PESI) score to predict the outcome variable of death within 30 days. Validation was performed in an independent sample of 182 patients with active cancer and PE.

Results-POMPE-C included eight predictors: body mass, heart rate $>100$, respiratory rate, $\mathrm{SaO} 2 \%$, respiratory distress, altered mental status, do not resuscitate status, and unilateral limb

\footnotetext{
(C) 2012 Elsevier Ltd. All rights reserved.

Correspondence to: Jeffrey A. Kline.

jkline@carolinas.org.

Publisher's Disclaimer: This is a PDF file of an unedited manuscript that has been accepted for publication. As a service to our customers we are providing this early version of the manuscript. The manuscript will undergo copyediting, typesetting, and review of the resulting proof before it is published in its final citable form. Please note that during the production process errors may be discovered which could affect the content, and all legal disclaimers that apply to the journal pertain.

Author Contributions: JAK conceived and designed the study, collected data, performed primary analysis and wrote the manuscript first draft PMR, AEJ and AP collected data and edited the manuscript DMC contributed to study design and collected data and edited the manuscript CVP assisted in study design, collected data, performed primary analysis edited the manuscript

COI summary: JAK has received research grant funding from AHRQ, NIH, Genentech, Pfizer and Octapharma within the past 3 years and owns stock in a medical device company, CP Diagnostics LLC and is an inventor on a patent pending involving the subject matter. CVP: Consultant to sanofi-aventis; speaker for sanofi-aventis; research funding from sanofi-aventis; research funding (current work) from GlaxoSmithKline

No other authors have any COI to disclose
} 
swelling. In the derivation set, the area under the ROC curve for POMPE-C was 0.84 (95\% CI: $0.82-0.87)$, significantly greater than PESI $(0.68,0.60-0.76)$. In the validation sample, POMPE-C had an AUC of 0.86 (0.78-0.93). No patient with POMPE-C estimate $5 \%$ died within 30 days $(0 / 50,0-7 \%)$, whereas $10 / 13(77 \%, 46-95 \%)$ with POMPE-C estimate $>50 \%$ died within 30 days.

Conclusion-In patients with active cancer and PE, POMPE-C demonstrated good prognostic accuracy for 30 day mortality and better performance than PESI. If validated in a large sample, POMPE-C may provide a quantitative basis to decide treatment options for PE discovered during cancer staging and with advanced cancer.

\section{Keywords}

Pulmonary embolism; outpatient; risk-stratification; Risk stratification; malignancy; prognosis; decision rule; treatment

\section{INTRODUCTION}

Risk stratification of acute pulmonary embolism (PE) generally refers to the use of objective criteria to predict the probability of clinical deterioration or death during a short-term follow-up period after diagnosis. The majority of published literature focuses on categorizing PE patients as low, moderate, or high risk of death, respiratory failure, or circulatory failure during the inpatient period or up to 30 days. ${ }^{1 ; 2}$ Methods of PE risk stratification include echocardiography, biomarkers, and scoring systems comprising clinical variables obtained from history and physical examination. In the emergency department setting, the category of risk can provide an objective basis to decide how and where to treat the patient. ${ }^{1-4}$ Patients with active malignancy represent a subgroup at particularly high risk both of developing PE and of having an increased risk of death after diagnosis of PE, when compared with patients who have no history of cancer or a past medical history of cancer in remission at the time of PE diagnosis. ${ }^{5-12}$ Patients with cancer and their oncologists also face the conundrum of how and where to treat unsuspected PE that is incidentally discovered on approximately $2 \%$ of computerized tomography pulmonary angiography scans ordered for the purpose of cancer staging or surveillance. ${ }^{13 ; 14}$ It can be reasonably hypothesized that many cancer patients with these incidentally discovered PE desire to be treated at home, but current literature lacks a decision tool to quantify the short-term prognosis for patients with active cancer and PE. ${ }^{15}$ The most widely validated scoring system for PE is the PESI tool, and a recent randomized trial demonstrated equivalent outcomes for patients with a low-risk PESI score randomized to have treatment initiated in the outpatient setting compared with the inpatient setting. ${ }^{16}$ However, PESI has not been tested specifically in patients with active cancer or PE. The objective of this study was to derive a multivariate, quantitative decision tool to assess the probability of death within 30 days among patients with active cancer who present in the outpatient environment with PE, compare its performance to PESI, and to perform initial validation studies of its prognostic accuracy using four independent samples of patients with active cancer and PE and to create a web-based electronic userform of the model for future research use.

\section{METHODS}

The prediction instrument was derived as a preplanned secondary analysis of the multicenter EMPEROR (Emergency Medicine Pulmonary Embolism in the Real World) Registry dataset, which was collected in 22 community and academic emergency departments in the United States, from January 1, 2005 until December 29, 2008. The methods of data collection have been reported elsewhere. ${ }^{11}$ Briefly, participating sites in EMPEROR prospectively collected 264 data elements on 1,880 patients diagnosed with PE in the 
emergency department (ED), including 30 day outcomes. The data collection instrument was designed to collect detail about risk factors for PE as well as data known to predict adverse outcomes. The form included demographic data, vital signs, comorbidities, risk factors for thrombosis, values of biomarkers including troponins and natriuretic peptides, echocardiographic results, and radiographic results. For many of these elements, additional detail was collected. Importantly, the malignancy variable was further divided into active cancer (defined as metastatic disease or cancer under the care of an oncologist), metastatic malignancy, or inactive cancer (defined as in remission by the patient and no longer under the active care of an oncologist). That level of detail was collected in response to multiple studies that found that active cancer, but not cancer in remission, is a risk factor for death from PE. ${ }^{5-12}$ We determined which patients with active cancer and PE discovered on imaging performed for other reasons such as staging or cancer surveillance using a retrospective chart review for the explicit definition of PE discovered on a CT scan done for initial staging of newly diagnosed cancer. In this subanalysis, we did not include patients who had a CT scan done to evaluate for possible worsening of known cancer, or abdominal CT scans with intravenous contrast that showed a pulmonary vascular filling defects in slices incidentally found in the lower lung zones. For a separate subanalysis, we also identified patients who had PE diagnosed in the ED and were discharged from the ED to home. Patients were followed prospectively for in-hospital adverse outcomes and for survival until 30 days after the ED visit.

\section{Selection of predictor variables}

The selection of variables, their analysis, model construction, goodness of fit, and validation were performed in accordance with published standards. ${ }^{17}$ We first conducted a comprehensive review of published studies that reported significant predictors of adverse outcome of PE. ${ }^{15}$ This review suggested 24 potential independent variables (appendix). The dependent variable was mortality at 30 days. The 24 potential predictor variables were screened for inclusion in the multivariate model using two methods: 1 . Data were first analyzed with classification and regression technique (CART, Salford Systems Inc, San Diego, CA) ${ }^{18} ; 2$. Bivariate analysis was then performed to screen for potentially important differences in means (using unpaired t-test) or frequency (using Chi square) for the 24 variables between survivors and decedents with variables. For a variables to move forward to the multivariate testing state, the variable had to have both a relative weight $>0$ from CART and $\mathrm{P}<0.1$ from bivariate analysis.. Retained continuous predictor variables (e.g., heart rate) underwent receiver operating characteristic (ROC) curve analysis to determine the cutoff corresponding to the lowest likelihood ratio negative for prediction of death at 30 days.

\section{Derivation of the multivariate model}

Retained predictors were then entered into a second logistic regression analysis, with the independent predictor being death at 30 days. Model fit was assessed by $\mathrm{P}$ values from the Pearson's goodness of fit Chi-Square, Hosmer Lemeshow test, the Pseudo (McFadden) Rsquare and area under the ROC curve in the derivation dataset (C statistic). 19;20

\section{Preliminary validation of the model}

We tested the prognostic accuracy of the derived instrument in six prospectively collected samples of patients diagnosed with PE in the ED. These studies included one multicenter sample from the US, ${ }^{21}$ three single-center samples from the US, ${ }^{22 ; 23}$ one single center registry from New Zealand and one multicenter sample from Europe. ${ }^{24}$ The clinical data for these samples were all collected prospectively by qualified research associates who had standardized training in data definitions. Data came from the most accurate source or combination of sources which included the patient in real time, the patient's primary 
physician, the emergency care providers and the medical record. These databases were reduced to patients with active cancer and PE, and the percentage mortality estimate (i.e., the predicted probability of death within 30 days) was then determined by taking the antilog and solving the logistic regression equation for the $\mathrm{P}$ term. Using a criterion standard of 30 day all-cause mortality, prognostic accuracy was assessed from the area under the curve (AUC) for the ROC curve, with the assumption that lower limit $95 \%$ confidence interval for the AUC greater than 0.5 indicated potential validity. We also calculated the PESI score to all 1880 patients with PE in the derivation dataset and then for the 408 patients with PE and active cancer, and compared its prognostic accuracy to POMPE-C based upon AUC under the ROC and the percentage of the sample with a low risk PESI score ( $<66$ points) and the 30 day rate of death in this low risk group.

\section{Creation of web-based prototype}

The validated model was then converted to an electronic form by creating an input form in Visual Basic designed to explain and capture each variable from the user and output the numeric probability of death using the derived and validated logit equation. The form was then uploaded to a server and a universal resource locator address assigned to allow public access.

\section{RESULTS}

\section{Candidate variable selection}

To assess the potential significance of all 25 predictor variables, the original EMPEROR database $(\mathrm{N}=1,880)$ was reduced to patients with active cancer and $\mathrm{PE}$, excluding patients who had comfort care status only. This process excluded 1,346 patients with no history of cancer, 108 who had a history of cancer thought to be in remission, and 18 patients with active cancer and comfort care only status, leaving 408 patients with active cancer, of whom $51(12.5 \%)$ died within 30 days of any cause. Table 1 lists the means and frequency data for the 25 predictor variables for survivors compared with patients who died for the 408 patients with active cancer and PE. Table 1 also shows the relative weights of each predictor determined from CART and the bivariate P values. Eight variables had a non-zero weight from CART and a $\mathrm{P}<0.1$ from either the unpaired t-test or Chi-Square and were thus retained for the multivariate model.

\section{Derivation of the multivariate model}

The retained eight variables were: 1 . Body weight, 2. Respiratory rate, 3. SaO2\%, 4. Heart rate over 99 beats/min, 5. Altered mental status, 6. Respiratory distress, 7. Do not resuscitate status, 8. Unilateral limb swelling. The first three variables are entered as continuous data and the last five variables are entered as dichotomous values. These variables and their definitions are presented in Table 2. In logistic regression analysis, all 8 variables were significant predictors of death, as demonstrated by the lower limit of the $95 \%$ confidence interval for the odds ratios associated with each variable (Table 2). The Pearson Chi-square goodness of fit P value was 0.94 , the Hosmer Lemeshow P value was 0.48 , the McFaddens' $\mathrm{R}^{2}$ was 0.21 . When the model was applied to the 408 patients with active cancer and PE in the derivation dataset, the AUC under the ROC was 0.84 (95\% CI: 0.78 to 0.89). When applied to the entire dataset of 1880 patients with PE, the model had an AUC for the ROC of $0.80(0.76$ to 0.85$)$. When applied to the 108 patients with a history of inactive cancer, the derived model had an AUC of 0.85 but because only seven of these 108 patients died within 30 days, the confidence intervals were wide (0.66 to 1.0). Taken together, these data indicate reasonable model fitness and predictive accuracy in the derivation set of patients with cancer and PE. ${ }^{19 ; 20}$ 


\section{Prognostic accuracy of PESI}

When applied to the entire 1880 patients in the derivation sample with PE, the PESI score demonstrated good overall diagnostic accuracy, with an AUC of 0.79 (0.75 to 0.83), and $610 / 1880$ patients (34\%) had PESI $<66$, and only 5/610 with PESI $<66$ died within 30 days, confirming the good overall performance of PESI as tool to identify low-risk patients with $\mathrm{PE}$ in the heterogenous derivation sample. However, when PESI was applied to the 408 patients with active cancer and PE, the AUC was significantly reduced to 0.68 ( 0.60 to 0.76 ) and only 3 patients $(0.8 \%)$ had PESI $<66$. None of these three patients died within 30 days. Examination of higher cutoffs for PESI to allow a larger fraction of the population to be deemed low risk caused an unfavorable increase of inclusion of patients who died within 30 days. For example, 111/408 patients (27\%) with active cancer and PE had a PESI score $<100$, and $8 / 111(7 \%)$ died within 30 days.

\section{Preliminary validation of the model}

The six validation samples contained a total of 14,121 patients, of whom 202 had active cancer and PE+. The database from Europe also did not explicitly record the terms for altered mental status or do not resuscitate but in accordance with original inclusion and exclusion requirements for study enrollment, these were assumed to be absent for all patients. Data were missing [body mass (12), respiratory rate (5) and pulse oximetry (3)] in 20 patients, seven from the European sample and three from the New Zealand sample and these 20 patients were excluded. Thus, 182 patients had complete data, of whom, 27 died within 30 days (mortality 15\%, 95\% CI: 10 to $21 \%$ ). Table 3 presents the mean percentage mortality estimate for patients from the individual studies comprising the total validation sample, stratified by survival status at 30 days. Figure 1 shows the ROC curve derived in the aggregated sample of all 182 validation patients. The area under the curve was 0.86 (95\% CI: 0.78 to 0.93 ) indicating good overall discriminatory value in the validation sample. Figure 2 shows a frequency dot plot of each probability estimate, stratified by survival status at 30 days.

The percentage estimates of mortality from the logistic equation were examined for cutoffs that predicted a very low and very high probability of death within 30 days for each patient in the validation sample. The cutoff of 5\% produced a very low probability of death. In the validation sample, 50 patients $(27 \%)$ of patients in the validation set with a mortality estimate of 5\% or less died within 30 days. Stated another way, all patients who died had a mortality estimate $>5 \%$, indicating a sensitivity of $27 / 27$ (100\%, 95\% CI: 88 to $100 \%)$ and 106 patients who survived $>30$ days had a mortality estimate $>5 \%$ (specificity $50 / 156=$ $32 \%, 95 \%$ CI: 25 to $40 \%$ ), producing a posterior probability of death equal to 0/50 (95\% CI: 0 to $7 \%$ ). The cutoff of $50 \%$ produced a very high probability of death; 13 patients had a mortality estimate $>50 \%$, and 10/13 died (posterior probability $73 \%, 95 \%$ CI: 46 to $95 \%$ ).

From both the derivation set and the validation set, we identified 16 patients whom we confirmed to have PE discovered incidentally, of whom none ( $0 \%)$ were discharged from the emergency department and one died within 30 days, but after hospital discharge. The mean POMPE-C score for the 15 patients who survived was $5 \pm 5 \%$, and 9 of 15 had a score $<5 \%$. The patient who died had a POMPE-C score of $46 \%$ and was the only patient with a do not resuscitate order. We also identified 19 other patients who were discharged from the ED, none of whom died within 30 days. The mean POMPE-C for these patients was $6 \pm 8 \%$ and 14 of 19 had a score $5 \%$.

\section{Web-based prototype}

The electronic input form was programmed to collect and transform the five dichotomous and three continuous variables into a numeric probability $(\mathrm{P})$ of death using the formula $\mathrm{P}=$ 
$100 *(1-1 /(1+\operatorname{Exp}(3.718+$ DNR $* 1.551+$ RespDist $* 0.800+$ ULS $* 0.734+$ AMS $* 1.473$

$+\mathrm{HR}+(\mathrm{RR} * 0.044)+\left(\mathrm{Sao}^{*} *-0.063\right)+($ bodyweight $\left.\left.\left.*-0.012)\right)\right)\right)$. The resultant prototype

device can be tested at http://www.studymaker.com/projects/pompe/index.php

\section{DISCUSSION}

We derived POMPE-C to address an unmet need for an accurate prognostic tool in patients with acute PE and active cancer. The POMPE-C tool demonstrated good overall diagnostic accuracy in both the derivation dataset (area under the receiver operating characteristic curve $=0.84,95 \%$ CI: 0.78 to 0.89 ) and the validation sample (area $=0.86,0.78$ to 0.93 ) of patients with PE and active cancer. ${ }^{25}$ Moreover, in the validation sample, 27\% $(\mathrm{n}=50)$ of patients had a POMPE-C estimate $\mathbf{5} \%$, and none of these 50 died within 30 days $(0 / 50$, 95\% CI: 0 to $7 \%$ ), suggesting clinical utility to the 5\% threshold to define low risk in a future clinical trial designed to increase home treatment of $\mathrm{PE}$ for cancer patients. We chose the 30 day mortality endpoint because this is the primary endpoint for the PESI score, and Aujesky et al has demonstrated that patients with PESI $<66$ generally have good outcomes when treated as outpatients. In patients with active cancer, the prognostic accuracy of the PESI was significantly degraded when compared with its accuracy in the whole derivation population, and PESI demonstrated a significantly lower area under the receiver operating characteristic curve than POMPE-C in the active cancer subgroup. Only 3/408 (0.8\%) of patients with active cancer and PE had a low risk PESI score ( $<66$ points), and raising the low-risk PESI threshold to 100 , such that $27 \%$ of the derivation group was considered low risk, led to an unacceptable $7 \%$ death rate at 30 days. Taken together these data indicate that POMPE-C can accurately prognose PE in patients with active cancer, and indicate that POMPE-C may be more accurate than PESI for this goal.

A primary motivation of this study stems from the need for a validated clinical pathway in the management of cancer patients who, in undergoing staging imaging that includes contrast-enhanced pulmonary vasculature imaging, are incidentally discovered to have PE. Prior work has suggested that this occurs in approximately $1.5-2.5 \%$ of such scans. ${ }^{13 ; 14}$ Published evidence suggests that not all incidental clots are benign, especially in the case of larger clots. ${ }^{26 ; 27}$ Following the work of Aujesky et al, the goal of this effort is to use POMPE-C in a clinical trial of outpatient management for low risk patients with active cancer and PE, including those with incidentally discovered PE, and with a POMPE-C mortality estimate of $5 \%$ or less. ${ }^{16}$ Additionally, for selected patients with advanced cancer and PE, an accurate quantitative prediction instrument may better align the care patients want with the care they receive near the end of life.

The scope and selection of prediction variables warrants specific consideration. First, the data the EMPEROR registry was designed to include including specific variables relevant to prognosis of cancer patients with $\mathrm{PE}$. We used a rigorous data mining process, including the combined use of stochastic methods and classification and regression to enhance the sensitivity and specificity of the final model. ${ }^{28}$ This process resulted in the selection of eight predictor variables, five of which we submit are fairly well recognized general predictors of outcome from $\mathrm{PE}$ (heart rate, respiratory rate, $\mathrm{SaO} 2 \%$, respiratory distress, and altered mental status), as well as three predictors that appear to be more specific to patients with cancer and PE (body weight, do not resuscitate status, and unilateral limb swelling). Body mass probably serves as a very crude but reliable surrogate to the stage of cancer, and body mass was chosen over body mass index (BMI), because it is simpler and BMI did not improve the model fitness. The "do not resuscitate" (DNR) status can be viewed as both a predictive and deterministic variable. However, from a probabilistic standpoint, two facts remain true: 1 . When a patient with cancer is diagnosed with PE, the patient's DNR status can be determined as either positive or negative, and 2. The DNR status strongly influences 
outcome. Thus, we argue that a decision rule that fails to advantage itself of the predictive power of DNR status will also fail to be as accurate as is reasonably possible. Unilateral limb swelling probably reflects the worsened prognosis of concomitant DVT either in the leg or arm, the latter of which is often associated with an indwelling catheter, which may imply additional vulnerability such as ongoing chemotherapy. Several registries have demonstrated increased risk of death from PE with concomittant DVT and this factor is present in the Geneva risk score. ${ }^{29}$

The selected eight variables were entered into a logistic regression instead of a classification tree as the final model as the goal was to produce a numeric percentage probability estimate of death, and because logistic regression often produces better results in validation in studies of short term prognosis of acute cardiovascular disorders. ${ }^{30 ; 31}$ The resulting equation found all variables significant, and the final model demonstrated good fit in the derivation set, but most importantly had good accuracy in independent samples that included patients from multiple countries. Taken together, these methods should confer durable external validity to the model.

Limitations to this study include the lack of a large prospective validation sample, which will be the next research step. ${ }^{32}$ Neither EMPEROR nor any of the validation studies were designed to capture patients with incidentally discovered PE on staging CT scans.

Nonetheless, we identified 16 patients with PE discovered in this situation, as well as 19 other patients who were discharged from the ED, and POMPE-C estimated a low risk of death (i.e., 5\%) in two-thirds of these patients. The POMPE-C model lacks information about malignancy type and staging because they were not collected in the derivation sample. We believe these variables should be collected in a larger validation study and then tested in simulation analyses to determine if they can improve the already good diagnostic accuracy of POMPE-C. We defined active cancer using similar language employed by the Well's score for pretest probability assessment for PE, although we acknowledge that distinguishing active versus inactive cancer will inherently have some ambiguity especially in the ED setting. This concern is mitigated somewhat because the area under the ROC for POMPE-C was 0.85 for patients with inactive cancer, although the confidence intervals were very wide ( 0.66 to 1.0$)$. Some users may prefer to use an integer-based scoring system, derived from the coefficients taken from a logit equation that uses only dichotomized variables. However, in trial derivation, the process of dichotomizing respiratory rate, pulse oximetry, and body weight caused a loss of both precision and accuracy. The creation of a web-based userform fits into the larger initiative of many health care systems to convert medical records into an electronic format. Moreover, our prior published experience has suggested that emergency medicine clinicians frequently commit errors in their recall and use of decision rules for pulmonary embolism. ${ }^{33 ; 34}$

\section{Conclusion}

This study reports the derivation and initial validation of an eight variable quantitative decision rule that demonstrated good overall accuracy in predicting all-cause, near-term mortality for patients with active cancer and acute pulmonary embolism. Further validation in a larger sample of patients with active cancer and PE will be necessary.

\section{Acknowledgments}

Funding Support: NIH/NHLBI R01 HL074384 Glaxo Pharmaceuticals 


\section{Reference List}

1. Jaff MR, McMurtry MS, Archer SL, Cushman M, Goldenberg N, Goldhaber SZ, Jenkins JS, Kline JA, Michaels AD, Thistlethwaite P, Vedantham S, White RJ, Zierler BK. Management of Massive and Submassive Pulmonary Embolism, Iliofemoral Deep Vein Thrombosis, and Chronic Thromboembolic Pulmonary Hypertension: A Scientific Statement From the American Heart Association. Circulation. 2011; 1:1788-1830. [PubMed: 21422387]

2. Torbicki A, Perrier A, Konstantinides S, Agnelli G, Galie N, Pruszczyk P, Bengel F, Brady AJ, Ferreira D, Janssens U, Klepetko W, Mayer E, Remy-Jardin M, Bassand JP, Vahanian A, Camm J, De CR, Dean V, Dickstein K, Filippatos G, et al. Guidelines on the diagnosis and management of acute pulmonary embolism: the Task Force for the Diagnosis and Management of Acute Pulmonary Embolism of the European Society of Cardiology (ESC). Eur.Heart J. 2008; 29:2276-2315. [PubMed: 18757870]

3. British Thoracic Society Standards of Care Committee Pulmonary Embolism Guideline Development Group. British Thoracic Society guidelines for the management of suspected acute pulmonary embolism. Thorax. 2003; 58:470-483. [PubMed: 12775856]

4. Kearon C, Kahn SR, Agnelli G, Goldhaber S, Raskob GE, Comerota AJ. Antithrombotic therapy for venous thromboembolic disease: American College of Chest Physicians Evidence-Based Clinical Practice Guidelines (8th Edition). Chest. 2008; 133:454S-545S. [PubMed: 18574272]

5. Galanaud JP, Quenet S, Rivron-Guillot K, Quere I, Sanchez Munoz-Torrero JF, Tolosa C, Monreal M. Comparison of the clinical history of symptomatic isolated distal deep-vein thrombosis vs. proximal deep vein thrombosis in 11086 patients. J Thromb.Haemost. 2009; 7:2028-2034. [PubMed: 19793188]

6. Carson JL, Kelley MA, Duff A. The clinical course of pulmonary embolism. N Engl J Med. 1992; 326:1240-1245. [PubMed: 1560799]

7. Kasper W, Konstantinides S, Geibel A, Olschewski M, Heinrich F. Management strategies and determinants of outcome in acute major pulmonary embolism: Results of a multicenter registry. $\mathrm{J}$ Am Coll Cardiol. 1997; 30:1165-1171. [PubMed: 9350909]

8. Goldhaber SZ, Visani L, De Rosa M. Acute pulmonary embolism: Clinical outcomes in the international cooperative pulmonary embolism registry (ICOPER). Lancet. 1999; 353:1386-1389. [PubMed: 10227218]

9. Laporte S, Mismetti P, Decousus H, Uresandi F, Otero R, Lobo JL, Monreal M. Clinical predictors for fatal pulmonary embolism in 15,520 patients with venous thromboembolism: findings from the Registro Informatizado de la Enfermedad TromboEmbolica venosa (RIETE) Registry. Circulation. 2008; 117:1711-1716. [PubMed: 18347212]

10. Aujesky D, Obrosky DS, Stone RA, Auble TE, Perrier A, Cornuz J, Roy PM, Fine MJ. Derivation and validation of a prognostic model for pulmonary embolism. Am.J.Respir.Crit.Care Med. 2005; 172:1041-1046. [PubMed: 16020800]

11. Pollack CV, Schreiber D, Goldhaber SZ, Slattery D, Fanikos J, O'Neil BJ, Thompson JR, Hiestand B, Briese BA, Pendleton RC, Miller CD, Kline JA. Clinical characteristics, management, and outcomes of patients diagnosed with acute pulmonary embolism in the emergency department Initial Report of EMPEROR (Multicenter Emergency Medicine Pulmonary Embolism in the Real World Registry). J Am Coll Cardiol. 2011; 57:700-706. [PubMed: 21292129]

12. Spencer FA, Goldberg RJ, Lessard D, Reed G, Emery C, Gore JM, Pacifico L, Weitz JI. Factors associated with adverse outcomes in outpatients presenting with pulmonary embolism: the Worcester Venous Thromboembolism Study. Circ Cardiovasc Qual Outcomes. 2010; 3:390-394. [PubMed: 20606125]

13. Gosselin MV, Rubin GD, Leung AN, Hunag J, Rizk NW. Unsuspected pulmonary embolism: Prospective detection on routine helical CT scans. Radiology. 1998; 208:209-215. [PubMed: 9646815]

14. Douma RA, Kok MG, Verberne LM, KAMPHUISEN PW, Buller HR. Incidental venous thromboembolism in cancer patients: prevalence and consequence. Thromb Res. 2010; 125:e306e309. [PubMed: 20223502]

15. Kline JA, Miller DW. Risk stratification for acute pulmonary embolism. J Natl Compr Canc Netw. In press. 
16. Aujesky D, Roy PM, Verschuren F, Righini M, Osterwalder J, Egloff M, Renaud B, Verhamme P, Stone RA, Legall C, Sanchez O, Pugh NA, N'gako A, Cornuz J, Hugli O, Beer HJ, Perrier A, Fine MJ, Yealy DM. Outpatient versus inpatient treatment for patients with acute pulmonary embolism: an international, open-label, randomised, non-inferiority trial. Lancet. 2011; 378:41-48. [PubMed: 21703676]

17. Moss M, Wellman DA, Cotsonis GA. An appraisal of multivariable logistic models in the pulmonary and critical care literature. Chest. 2003; 123:923-928. [PubMed: 12628895]

18. Kline JA, Johnson CL, Pollack CV, Diercks DB, Newgard CD, Hollander JE. Pretest probability assessment derived from attribute matching. BMC Medical Informatics and Decision Making 2004. 2005; 5:26-37.

19. Kuss O. Global goodness-of-fit tests in logistic regression with sparse data. Stat.Med. 2002; 21:3789-3801. [PubMed: 12483767]

20. Hosmer DW, Hosmer T, Le CS, Lemeshow S. A comparison of goodness-of-fit tests for the logistic regression model. Stat.Med. 1997; 16:965-980. [PubMed: 9160492]

21. Courtney DM, Kline JA, KABRHEL C, Moore CL, Smithline HA, Nordenholz KE, Richman PB, Plewa MC. Clinical features from the history and physical examination that predict the presence or absence of pulmonary embolism in symptomatic emergency department patients: Results of a prospective, multicenter study. Ann.Emerg Med. 2010; 55:305-315.

22. Kline JA, Hernandez J, Rose G, Norton HJ, Camargo CA Jr. Surrogate markers for adverse outcomes in normotensive patients with pulmonary embolism. Crit Care Med. 2006; 34:27732180. [PubMed: 16943732]

23. Kline JA, Hogg M. Measurement of expired carbon dioxide, oxygen and volume in conjunction with pretest probability estimation as a method to diagnose and exclude pulmonary venous thromboembolism. Clin Physiol Funct.Imaging. 2006; 26:212-219. [PubMed: 16836693]

24. Roy PM, Durieux P, Gillaizeau F, Legall C, rmand-Perroux A, Martino L, Hachelaf M, Dubart AE, Schmidt J, Cristiano M, Chretien JM, Perrier A, Meyer G. A computerized handheld decisionsupport system to improve pulmonary embolism diagnosis: a randomized trial. Ann.Intern.Med. 2009; 151:677-686. [PubMed: 19920268]

25. Hanley JA, McNeil BJ. The meaning and use of the area under the receiver operating characteristic (ROC) curve. Radiology. 1982; 143:29-36. [PubMed: 7063747]

26. O'Connell C, Razavi P, Ghalichi M, Boyle S, Vasan S, Mark L, Caton A, Duddalwar V, Boswell W, Grabow K, Liebman HA. Unsuspected pulmonary emboli adversely impact survival in patients with cancer undergoing routine staging multi-row detector computed tomography scanning. J Thromb Haemost. 2011; 9:305-311. [PubMed: 20955348]

27. O'Connell CL, Boswell WD, Duddalwar V, Caton A, Mark LS, Vigen C, Liebman HA. Unsuspected pulmonary emboli in cancer patients: clinical correlates and relevance. J Clin Oncol. 2006; 24:4928-4932. [PubMed: 17050877]

28. Harrell FE Jr. Lee KL, Mark DB. Multivariable prognostic models: issues in developing models, evaluating assumptions and adequacy, and measuring and reducing errors. Stat.Med. 1996; 15:361-387. [PubMed: 8668867]

29. Wicki J, Perrier A, Perneger T, Bounameaux H, Junod A. Predicting adverse outcome in patients with acute pulmonary embolism: A risk score. Thromb Haemost. 2000; 84:548-552. [PubMed: 11057848]

30. Austin PC. A comparison of regression trees, logistic regression, generalized additive models, and multivariate adaptive regression splines for predicting AMI mortality. Stat.Med. 2007; 26:29372957. [PubMed: 17186501]

31. Austin PC, Tu JV, Lee DS. Logistic regression had superior performance compared with regression trees for predicting in-hospital mortality in patients hospitalized with heart failure. $\mathrm{J}$ Clin.Epidemiol. 2010; 63:1145-1155. [PubMed: 20304609]

32. McGinn TG, Guyatt GH, Wyer PC, Naylor CD, Stiell IG, Richardson WS. Users' guides to the medical literature: XXII: How to use articles about clinical decision rules. Evidence-Based Medicine Working Group. JAMA. 2000; 284:79-84. [PubMed: 10872017] 
33. Runyon MS, Richman PB, Kline JA. Emergency medicine practitioner knowledge and use of decision rules for the evaluation of patients with suspected pulmonary embolism: variations by practice setting and training level. Acad Emerg Med. 2007; 14:53-57. [PubMed: 17119186]

34. Kline JA, Peterson CE, Steuerwald MT. Prospective evaluation of real-time use of the pulmonary embolism rule-out criteria in an academic emergency department. Acad Emerg Med. 2010; 17:1016-1019. [PubMed: 20836787] 


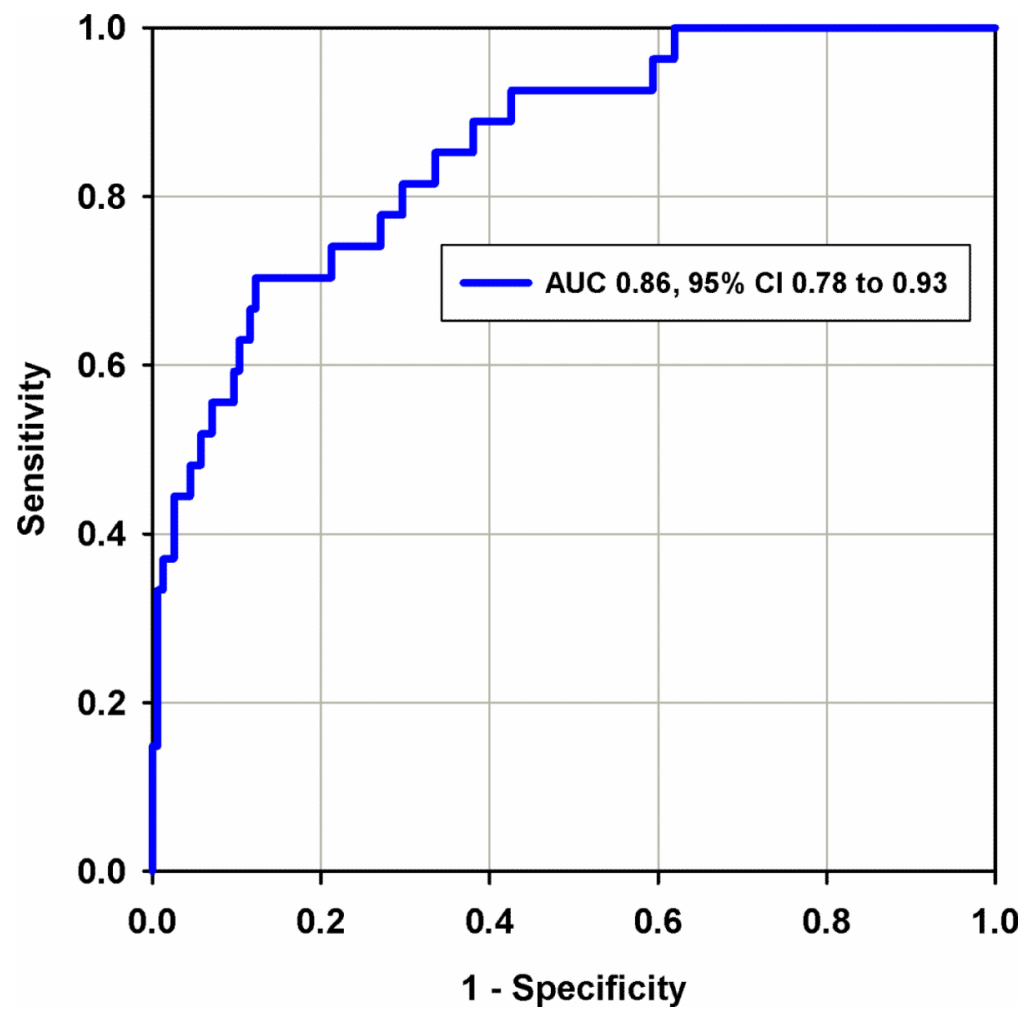

Figure 1.

Receiver operating characteristic curve using the percentage probability estimate of mortality from the logistic regression equation as the diagnostic test, and the outcome was 30 day mortality in the validation sample. 


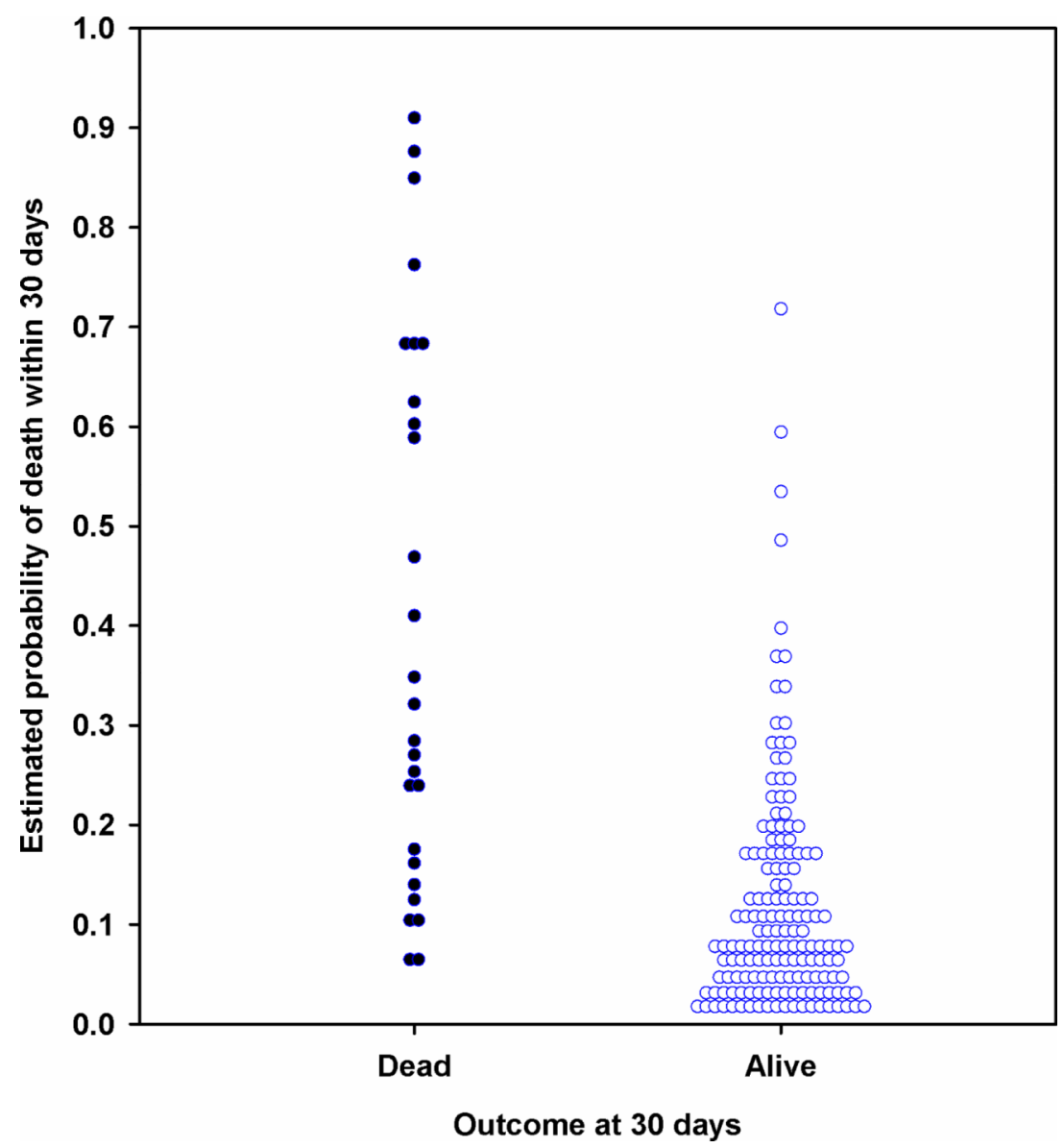

Figure 2.

Dot plot of the estimated probability of death from the logistic regression equation ( $\mathrm{Y}$ axis), stratified according to survival status at 30 days ( $\mathrm{X}$ axis). 

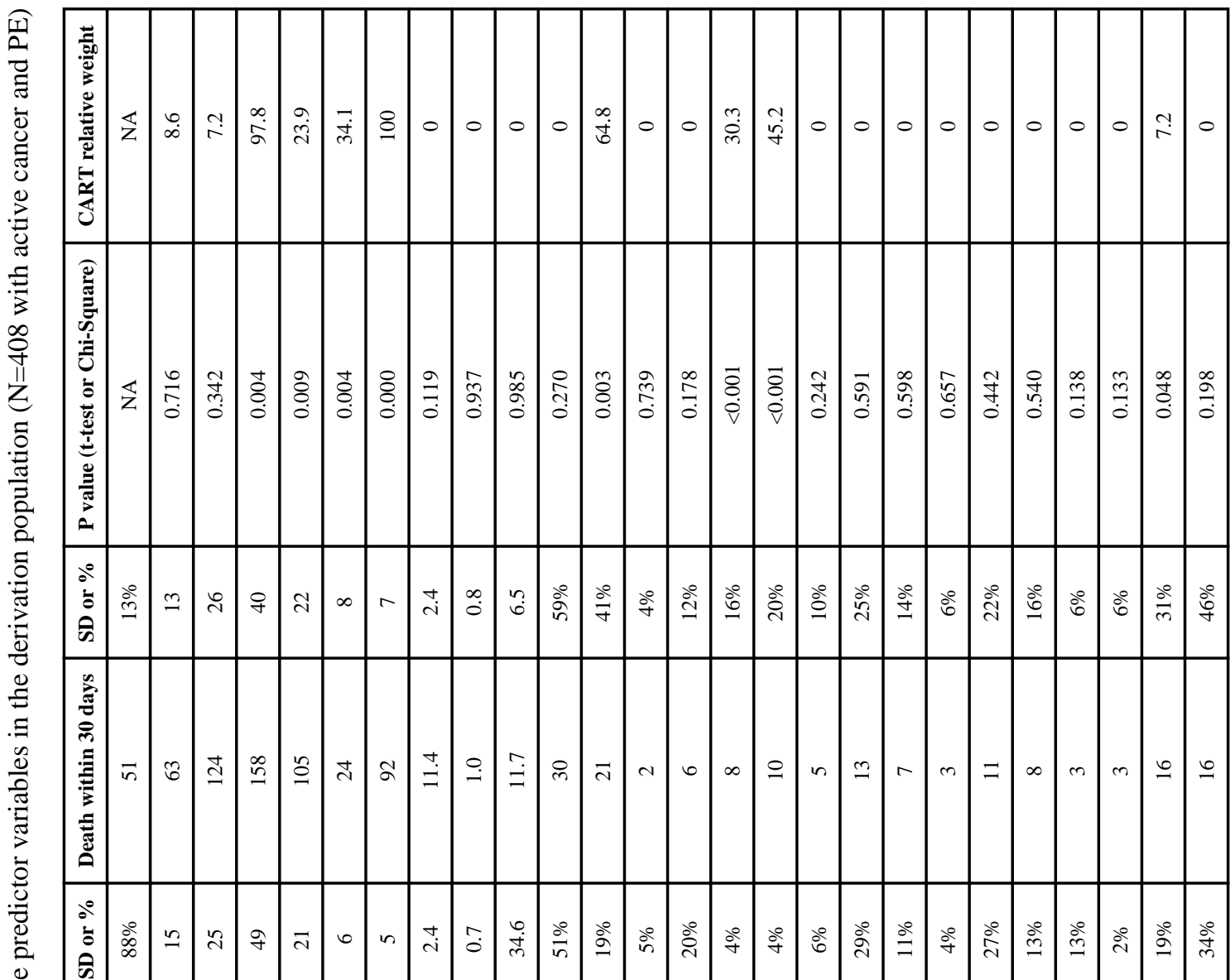

苟

ซ

$\frac{\infty}{2}$ 齐 
Table 2

Odds ratios for the eight predictor variables from logistic regression

\begin{tabular}{|c|c|c|c|}
\hline Variable & Coefficient & \multirow{2}{*}{ Odds Ratio } & \multirow{2}{*}{ Bias corrected 95\% CI } \\
\hline Intercept & 3.718 & & \\
\hline Patient weight $(\mathrm{lb})^{1}$ & 1.551 & 0.99 & 0.98 to 1 \\
\hline Highest respiratory rate (breaths $/ \mathrm{min})^{2}$ & 0.7996 & 1.05 & 1.01 to 1.09 \\
\hline $\mathrm{SaO} 2(\%)^{3}$ & 0.734 & 0.94 & 0.89 to 0.99 \\
\hline Heart rate $>99$ beats $/ \mathrm{min}^{4}$ & 1.473 & 2.8 & 1.38 to 5.64 \\
\hline Altered mental status 5 & 1.027 & 4.36 & 1.47 to 13 \\
\hline Respiratory distress ${ }^{6}$ & 0.044 & 2.22 & 1.12 to 4.43 \\
\hline Do not resuscitate status ${ }^{7}$ & -0.063 & 4.72 & 1.75 to 12.76 \\
\hline Unilateral limb swelling 8 & -0.0116 & 2.08 & 1 to 4.36 \\
\hline
\end{tabular}

Variable definitions:

1 Body weight measured or by patient's estimate

2 Highest documented respiratory rate within previous six hours

3 Most recent pulse oximetry measured with patient breathing room air.

${ }^{4}$ Positive if heart rate documented over 99 beats/min in previous six hours.

5 Positive if patient has acute impairment in consciousness, new disorientation, delirium, or confusion

${ }^{6}$ Positive if the patient shows obvious anxiety from dyspnea or evidence of increased work of breathing

7 Positive for new or existing verbal or written desire of patient to not be resuscitated from death

8 Positive for either a leg or arm has new, presentlly noticeable swelling causing it to be asymmetrical compared with the contralateral limb. 


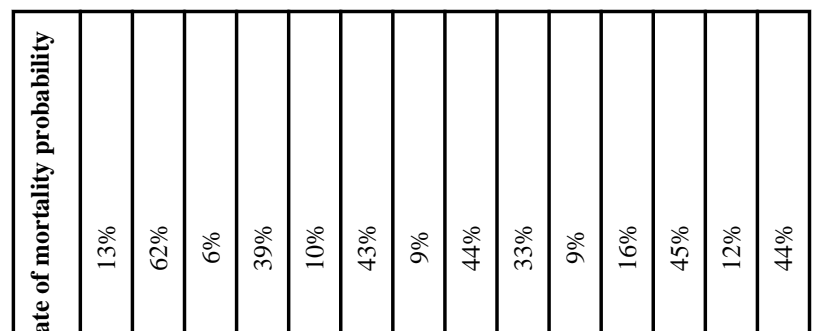

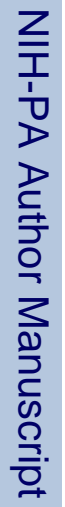

$m$
$\frac{1}{0}$
$\frac{0}{10}$

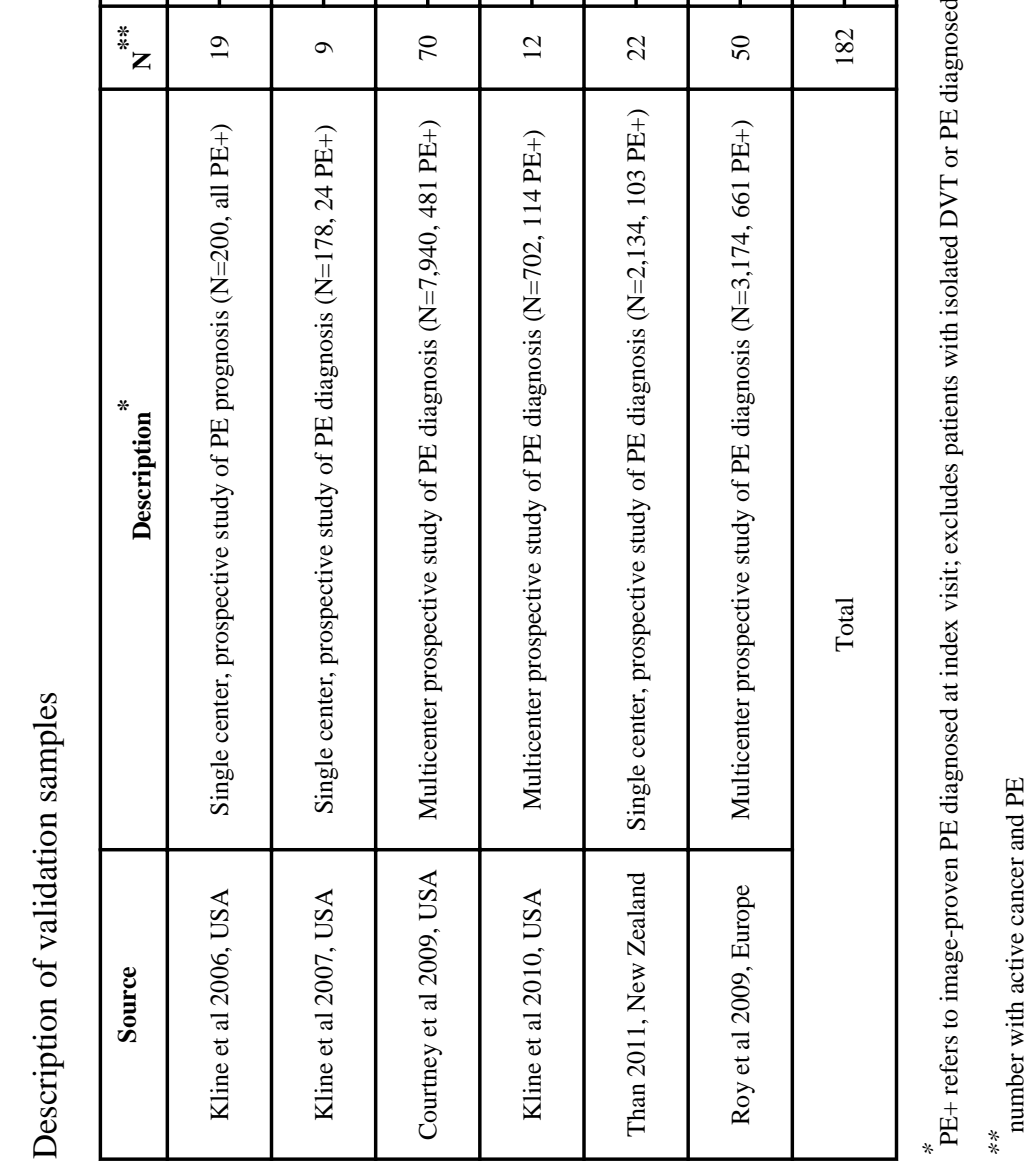

Thromb Res. Author manuscript; available in PMC 2013 May 01. 